\title{
Study on the Application of Large Tuned Mass Ratio in Damping System
}

\author{
Yeda Lianª, Qiang Ma ${ }^{\text {b, }}$, Yuqiang Zhang ${ }^{\mathrm{c}}$, R.M.Ali Washakh, \\ Yuanliang Zhang \\ School of Mechanics, Civil Engineering and Architecture, Northwestern Polytechnical University, \\ Xi'an 710129, China. \\ alianyeda@qq.com,, bmaqiang0331@163.com,'changyuqiang0616@163.com
}

Keywords: large tuned mass ratio; tuned mass damper; building damping.

\begin{abstract}
To solve the problem of insufficient bandwidth in damping frequency, it is the key to advance the application of tuned mass damping method in architectural design. This paper presents the idea that the tuned mass ratio increases the bandwidth of damping frequency. Based on deterministic excitation, the amplitude-frequency characteristics of TMD-building system are deduced theoretically and verify the validity of the large tuned mass ratio to increase the damping frequency. The relationship between the damping bandwidth and the tuned mass ratio is analyzed. At the same time, the research shows that the TMD is similar to the amplitude-frequency characteristic of the building structure under the condition of large tuned mass ratio, and the damage of TMD can be avoided in the design function.
\end{abstract}

\section{Introduction}

Tuned mass damper (abbreviated as TMD) can effectively reduce the wind-induced vibration response. Measured data show that TMD can reduce the wind-induced vibration response by more than 40\%. In recent years, TMD successful use in Taipei 101, Shanghai World Financial Center and some other landmarks further promote the tuned mass damping theory in the construction of structural design applications. However, the current "Building Seismic Design Code", has not yet included in the TMD design provisions, indicating that tuned mass damping technology in the field of building seismic design is not yet mature. A large number of research data shows that the seismic frequency components are more complex than the wind load, the existing TMD vibration frequency bandwidth cannot fully meet the needs of building damping, this is one of the reasons why TMD is difficult to apply to building damping ${ }^{[1]}$.

In a typical TMD study, considering the general building design of the ground carrying capacity and economic issues, the tuned mass ratio should be controlled within $5 \%$. With the giant structure of the controlled structure ${ }^{[2]}$, the giant frame suspension structure system ${ }^{[3]}$, the giant frame multifunction damping structure system ${ }^{[4]}$, the tuning frequency ratio can be greatly improved in value, or even more than $100 \%$. In this paper, a method to broaden the bandwidth of TMD operating frequency is proposed from the perspective of large harmonic frequency. Based on the theoretical deduction of the deterministic excitation, we analyzed the contribution of the large tuned mass ratio to the TMD frequency bandwidth, and studied the problem of building structure and TMD self-damping under the condition of large tuned mass ratio.

\section{Establishment of TMD - Building System Dynamic Analysis Equation}

Fig. 1 shows a schematic diagram of a building structure system with TMD installed. Where $m_{d}$ is TMD mass ratio, $K_{d}, C_{d}$ are the connection stiffness and damping between TMD and building structure, respectively. Building structural mass ratio and stiffness are $m_{l}, K_{l}$ and because of the small damping of building materials in actual engineering, the damping of building itself is not taken into consideration. $P_{l}(t), P_{d}(t)$ are acting on Building structure and TMD external excitation.

Defining $P_{1}(t)=P_{0} \sin (\omega t), P_{d}(t)=\lambda P_{0} \sin (\omega t), \lambda$ is the ratio of TMD to the external excitation of the building structure. 


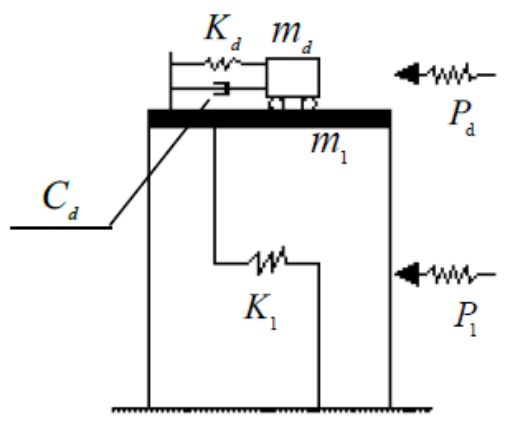

Fig.1 TMD-building system analysis model

The TMD-building system dynamic analysis equation is established as follows:

$$
\begin{aligned}
& m_{1} \ddot{x}_{1}+C_{d}\left(\dot{x}_{1}-\dot{x}_{d}\right)+\left(K_{1}+K_{d}\right) x_{1}-K_{d} x_{d}=P_{1}(t) \\
& m_{d} \ddot{x}_{d}+C_{d}\left(\dot{x}_{d}-\dot{x}_{1}\right)+K_{d}\left(x_{d}-x_{1}\right)=P_{d}(t)
\end{aligned}
$$

Where, $x_{1}, x_{d}$, respectively, is the building structure and TMD relative to the ground displacement, the other parameters defined in Fig.1. By means of numerical analysis, we can derive the structural displacement expression corresponding to the deterministic excitation. Then, other quantitative responses such as stress, strain, internal force and so on can be obtained indirectly. In order to obtain $x_{1}, x_{d}$, using transfer function solution. Defining the building structure, TMD transfer function are $\mathrm{E}$ and $\mathrm{F}$, then, the building structure and TMD dynamic response are:

$$
\begin{aligned}
& x_{1}(t)=H_{1}(\omega)\left(P_{1}(t)+P_{d}(t)\right)=H_{1}(\omega)(1+\lambda) P_{0} \sin (\omega t) \\
& x_{d}(t)=H_{d}(\omega)\left(P_{1}(t)+P_{d}(t)\right)=H_{d}(\omega)(1+\lambda) P_{0} \sin (\omega t)
\end{aligned}
$$

Substituting (2) into (1), the maximum value of the building structure and TMD displacement is:

$$
\begin{aligned}
& x_{1}=H_{1}(\omega)(1+\lambda) P_{0}=(1+\lambda) P_{0} \times \\
& \frac{1}{1+\lambda}\left\{\frac{(1+\lambda)^{2}\left(C_{d} \omega\right)^{2}+\left[m_{d} \omega^{2}-(1+\lambda) K_{d}\right]^{2}}{\left(m_{1} m_{d} \omega^{4}-m_{1} K_{d} \omega^{2}-m_{d}\left(K_{1}+K_{d}\right) \omega^{2}+K_{1} K_{d}\right)^{2}+\left(C_{d} \omega\right)^{2}\left(m_{1} \omega^{2}+m_{d} \omega^{2}-K_{1}\right)^{2}}\right\}^{0.5} \\
& x_{d}=H_{d}(\omega)(1+\lambda) P_{0}=(1+\lambda) P_{0} \times \\
& \frac{1}{1+\lambda}\left\{\frac{(1+\lambda)^{2}\left(C_{d} \omega\right)^{2}+\left[\lambda m_{1} \omega^{2}-(1+\lambda) K_{d}-\lambda K_{1}\right]^{2}}{\left(m_{1} m_{d} \omega^{4}-m_{1} K_{d} \omega^{2}-m_{d}\left(K_{1}+K_{d}\right) \omega^{2}+K_{1} K_{d}\right)^{2}+\left(C_{d} \omega\right)^{2}\left(m_{1} \omega^{2}+m_{d} \omega^{2}-K_{1}\right)^{2}}\right\}^{0.5}
\end{aligned}
$$

In order to express the physical meaning of the formula, the formulas (3) and (4) are internally extracted and replaced, and the results are as follows:

$$
\begin{gathered}
x_{1}=\frac{(1+\lambda) P_{0}}{K_{1}} \frac{1}{1+\lambda}\left\{\frac{(1+\lambda)^{2}\left(2 \zeta_{d} f h\right)^{2}+\left[h^{2}-(1+\lambda) f^{2}\right]^{2}}{\left[\mu f^{2} h^{2}-\left(h^{2}-1\right)\left(h^{2}-f^{2}\right)\right]^{2}+\left(2 \zeta_{d} f h\right)^{2}\left(h^{2}-1+\mu h^{2}\right)^{2}}\right\}^{0.5} \\
x_{d}=\left[\frac{(1+\lambda) P_{0}}{K_{1}}+\frac{\lambda P_{0}}{K_{d}}\right] \frac{1}{1+\lambda} \frac{(1+\lambda) f^{2}}{(1+\lambda) f^{2}+\frac{\lambda}{\mu}}\left\{\frac{(1+\lambda)^{2}\left(2 \zeta_{d} f h\right)^{2}+\left[\frac{\lambda}{\mu} h^{2}-(1+\lambda) f^{2}-\frac{\lambda}{\mu}\right]^{2}}{\left[\mu f^{2} h^{2}-\left(h^{2}-1\right)\left(h^{2}-f^{2}\right)^{2}\right]+\left(2 \zeta_{d} f h\right)^{2}\left(h^{2}-1+\mu h^{2}\right)}\right\}^{0.5}
\end{gathered}
$$

The dynamic amplification factors of building structure and TMD displacement are:

$$
A_{1}=\frac{1}{1+\lambda}\left\{\frac{(1+\lambda)^{2}\left(2 \zeta_{d} f h\right)^{2}+\left[h^{2}-(1+\lambda) f^{2}\right]^{2}}{\left[\mu f^{2} h^{2}-\left(h^{2}-1\right)\left(h^{2}-f^{2}\right)\right]^{2}+\left(2 \zeta_{d} f h\right)^{2}\left(h^{2}-1+\mu h^{2}\right)^{2}}\right\}^{0.5}
$$




$$
A_{d}=\frac{1}{1+\lambda} \frac{(1+\lambda) f^{2}}{(1+\lambda) f^{2}+\frac{\lambda}{\mu}}\left\{\frac{(1+\lambda)^{2}\left(2 \zeta_{d} f h\right)^{2}+\left[\frac{\lambda}{\mu} h^{2}-(1+\lambda) f^{2}-\frac{\lambda}{\mu}\right]^{2}}{\left[\mu f^{2} h^{2}-\left(h^{2}-1\right)\left(h^{2}-f^{2}\right)^{2}\right]+\left(2 \zeta_{d} f h\right)^{2}\left(h^{2}-1+\mu h^{2}\right)}\right\}^{0.5}
$$

The meanings of the symbols in the above formulas are as follows:

$\omega_{1} \ldots$ Natural frequency of the building structure, $\omega_{1}=\sqrt{K_{1} / m_{1}}$;

$\omega_{d} \_$TMD natural frequency, $\omega_{d}=\sqrt{K_{d} / m_{d}}$;

$\omega$ _External excitation frequency;

$f \ldots$ TMD and building structure frequency ratio $f=\omega_{d} / \omega_{1}$;

$h$ _ The frequency ratio of external excitation and building structure,

$h=\omega / \omega_{1}$;

$\mu \ldots$ TMD and building structure mass ratio, $\mu=m_{d} / m_{1}$.

\section{TMD-building system amplitude-frequency characteristics analysis}

Additional damping in the process of shock absorption has a certain energy consumption, in order to highlight the role of energy modulation damping, temporarily without considering the additional damping, let $\mathrm{C}_{\mathrm{d}}=0$. Then the formula (8) can be written as:

$$
\begin{aligned}
& A_{1}=\frac{1}{1+\lambda} \frac{(1+\lambda) f^{2}-h^{2}}{h^{4}-h^{2}\left[1+f^{2}(1+\mu)\right]+f^{2}} \\
& A_{d}=\frac{1}{1+\lambda} \frac{(1+\lambda) f^{2}}{(1+\lambda) f^{2}+\frac{\lambda}{\mu}} \frac{(1+\lambda) f^{2}+\frac{\lambda}{\mu}-\frac{\lambda h^{2}}{\mu}}{h^{4}-h^{2}\left[1+f^{2}(1+\mu)\right]+f^{2}}
\end{aligned}
$$

For building structures where TMD is not installed, $\mu, \lambda$, and $f$ in formula (9) are zero, respectively. Then the formula (9) can be changed to:

$$
A_{1}=\left|\frac{h^{2}}{h^{2}-h^{4}}\right|
$$

Drawing the building amplitude curve using formula (10) shown in Fig.2.

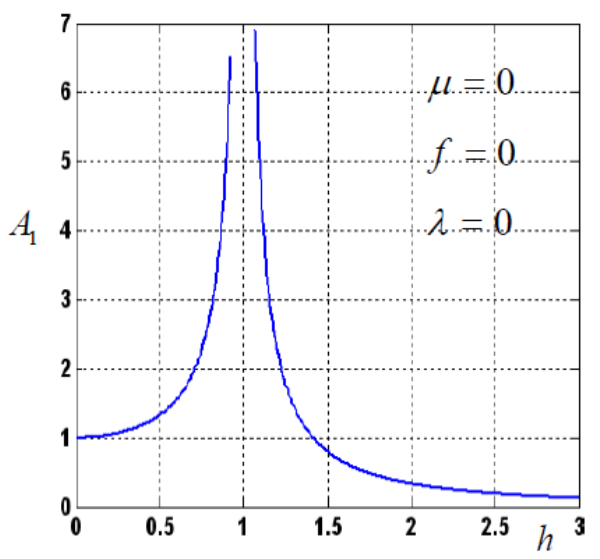

Fig.2 Building structure without TMD A1-h characteristic curve

When the external excitation frequency is equal to the natural frequency of the structure, that is to say, $h=1$, the response of the structure is infinite due to resonance, and it is consistent with the actual 
physical phenomena. On the one hand, the conclusion proves the reliability of the above theoretical deduction process; on the other hand, it reveals the breakthrough point of building vibration suppression.

In order to avoid interference with frequency ratio $f$ changes, we will take $f$ temporary value, let $f$ $=1$. In conventional TMD designs, the mass ratio $\mu$ is typically less than $5 \%$. As can be seen in Fig.3, the conventional TMD design has a very narrow damping frequency bandwidth, which explains why TMD is prone to failure in an earthquake.
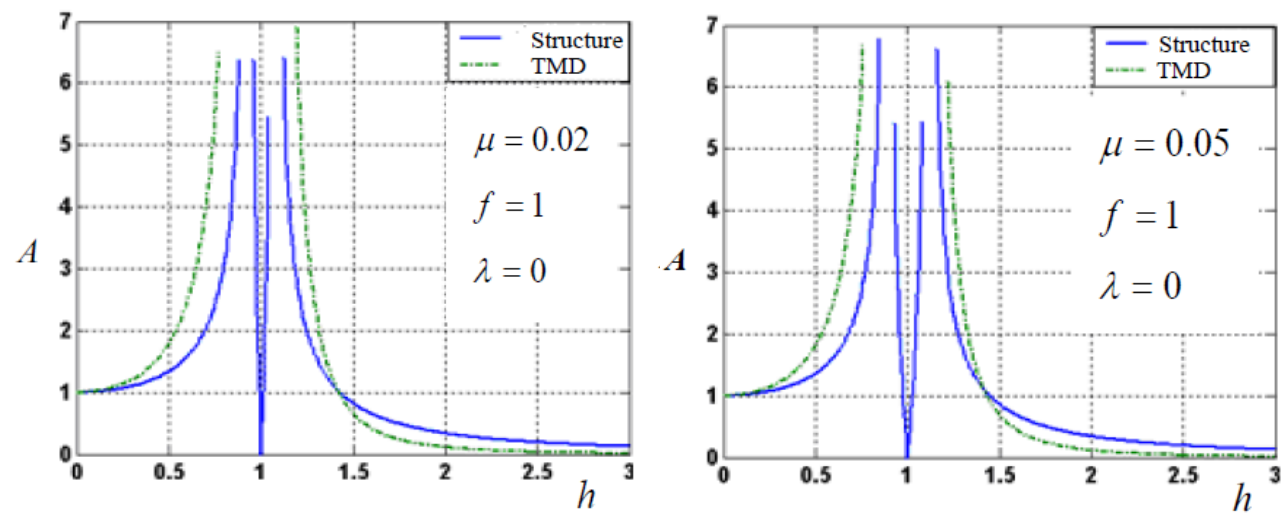

Fig.3 TMD building system A-h characteristic curve

In the new type of building system, such as the giant structure, the structure itself is designed to have the sub-structure of the tuned mass damping function, so as to construct the large tuned mass ratio. Fig.4 shows that the large frequency mass ratio can greatly widen the effective vibration frequency band of the resonance region and increase with the increase of $\mu$. In addition, compared with the results shown in Fig. 3 and Fig. 4, it can be found that the TMD is opposite to the amplitudefrequency characteristic of the building structure under the condition of conventional tuning mass ratio. That is, the TMD response will increase when the building structure response is controlled. The TMD is consistent with the amplitude-frequency characteristic of the building structure under the condition of large tuned mass ratio, which can effectively prevent the TMD falling off in the earthquake.
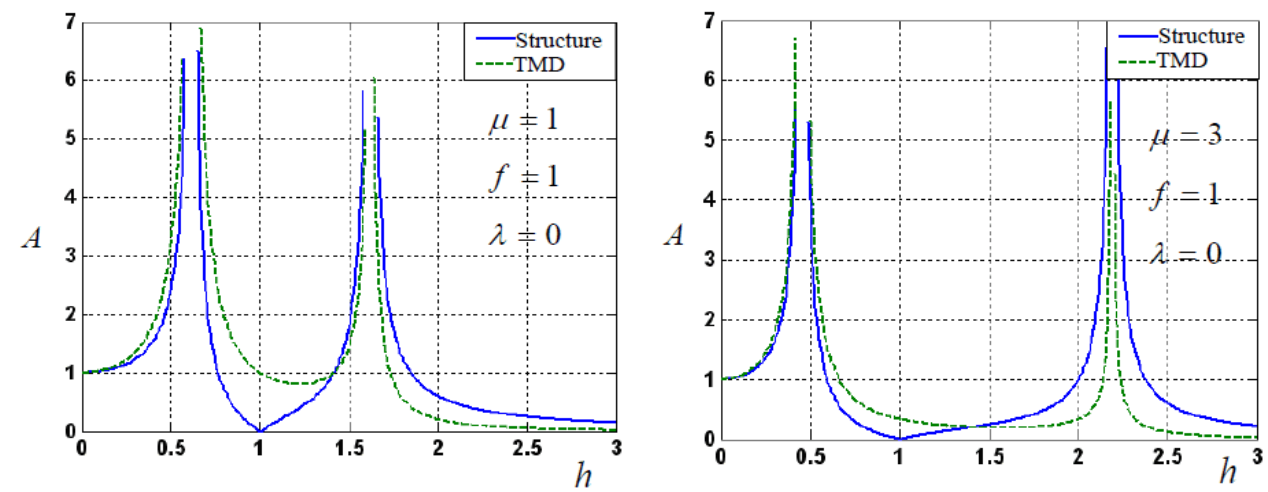

Fig.4 The A-h characteristic curve of building system under the condition of large tuned mass ratio

In the results shown in Fig. 3 and Fig. 4, the peak value of the resonance zone structure decreases and a new peak appears on both sides of the resonance region. This is because the theoretical calculation does not consider the additional damping effect, the actual TMD design should have additional damping, so there is no infinite peak.

\section{Conclusion}

Based on the theoretical derivation of the TMD-building system's amplitude-frequency characteristics, it is proved that the tuned mass ratio can increase the bandwidth of the tuned mass damping, and further improve the theoretical system of tuned mass damping, and expand the application of tuned mass damping. But through this study, it can be found that there are still some practical problems to be resolved: 
(1) In this paper, we study only the problem of frequency variation of frequency modulation, while the peak of the region response outside the damping bandwidth exists objectively. Additional damping can be further attenuated by energy dissipation, that is, weakening the peak, but the problem of additional damping optimization under the condition of large tuned mass ratio is still to be further studied.

(2) By combing the research status of the building structure system, it can be found that the large tuned mass ratio can be achieved in the mega frame structure design. But how to apply it in a broader architecture is a matter that needs further discussion, which may also be an aspect of the development of a new building structure system.

(3) In this paper, we use the deterministic excitation to carry out the dynamic analysis of TMDbuilding structure system. The purpose is to facilitate the theoretical derivation and prove the reliability of the theoretical derivation process. But in reality, the earthquake is a random process, and there are inevitably uncertainties in the architectural design and construction. Therefore, the large tuned mass ratio based on the reliability design issues need further study.

\section{Acknowledgments}

Supported by: National Natural Science Foundation of China Under Grant No. 51308458.

Biography: Yeda Lian (1980-),male, postdoctor, associate professor, major in seismic research of structures.

\section{References}

[1]. Feng M Q,MitaA. Vibration Control of Tall Building Using Mega-Sub Configuration [J].ASCE Journal of Engineering Mechanics, 1995,121(10):1082-1087.

[2]. Tao Li, Yeda Lian, Xun'an Zhang. Applying Double Modulation Model for Non-stationary Earthquake to Analyzing Seismic Response of MSCSS [J].Journal of Northwestern Polytechnical University, 2011,29(5) :709-714.

[3]. Yeda Lian, Xun'an Zhang, Zhaoxia Wang. Analyses of Vertical Earthquake Responses of the Mega-Beam Floor in the Vibration-Absorption Structure with Large Tuned Mass Ratio.[J] Industrial Construction, 2011,41(2):32-34.

[4]. Zongjian Lan, Yuji Tian. Structure-Control Mechanism of Multifunctional Vibration-Absorption of RC Megaframe Structures [J]. Industrial Construction,2002,32(1):4-6. 